\title{
27 \\ Androgenetic Alopecia, Trichotrophic Substances, and Histologic Studies of the Human Scalp
}

John T. Headington, MD

From the Department of Dermatology, The University of Michigan, School of Medicine, Ann Arbor. Michigan

\section{Androgenetic Alopecia}

The cosmetic effect of normal hair growth is a function of both hair shaft thickness and maximum obtainable length. Clinical androgenetic alopecia or "balding" is the result of progressive hypoplasia (miniaturization) of the terminal hair follicles of the scalp. 'The hair shafts produced by these small follicles decrease in diameter and shorten in length until they fall below an acceptable cosmetic threshold, ie, no longer visible.

An essential element in the development of androgenetic alopecia in both men and women is the sensitivity of individual follicles to normal amounts of circulating androgens. Androgens, particularly testosterone and its cellular metabolites, are required for the initiation and progression of follicular miniaturization. A second important requirement that dictates both the qualitative and quantitative extent of follicular miniaturization is the degree of penetrance of the gene or genes that determine where and to what degree the androgen-sensitive scalp follicle will produce shorter and thinner hairs.

During onset and development of androgenetic alopecia the density of follicular units (about one follicle per $\mathrm{mm}^{2}$ ) and the number of terminal follicles per follicular unit (about two to three) remain normal or near normal. ${ }^{1}$ It is possible that the number of hair follicles per unit area decreases with aging, a result of involutional or senescent alopecia superimposed on androgenetic alopecia. The presence of coexisting androgenetic and senescent alopecia in the same individual can be 
confusing to clinical investigators who evaluate the efficacy of trichotrophic substances in the treatment of androgenetic alopecia if the population under study includes individuals with senescent changes.

Androgenetic alopecia is characterized not only by follicular miniaturization but also by a shortened anagen phase. In both men and women with rapid-onset androgenetic alopecia, the "pull" or "tug" test is often positive for normal telogen hairs. Increased numbers of follicles in telogen can also be found in scalp biopsy samples from some patients with androgenetic alopecia who have positive "pull-tug" tests. By definition, this is a telogen effluvium even if localized only to the involved areas. The increased numbers of follicles in telogen that are found in androgenetic alopecia are a result of a shortened anagen time-in all probability, there is a shortened hair cycle.

Androgenetic alopecia, in terms of etiology and pathogenesis, is therefore clearly a variation in normal human physiology. By strict definition, I consider it to be a condition rather than a disease. The topical use of trichotrophic substances, such as minoxidil, that are intended to partially or completely reverse androgenetic alopecia must, as a consequence, continuously oppose a genetic program that is appropriate for the individual and must contend with any concomitant for aging.

\section{Trichotrophic Substances}

The action of any effective trichotrophic substances that induce hair follicle hypertrophy might, therefore, exploit the normal biologic potential for enlargement of the hair follicle, which can occur at any site. There appears to be no question that small hair follicles in many different sites can enlarge to form longer and thicker hairs. This "override" of what is anatomically normal for an area is the basis for local or generalized hirsutism and has been recognized by physicians for centuries. Probably the most common example of inappropriate follicular hypertrophy is manifested by the large hair follicles that colonize some nevocytic nevi, particularly the congenital forms. A partial list of other circumstances that result in follicular hypertrophy includes chronic irrita- tion, trauma, peripheral neuropathy, pretibial myxedema, certain neurodystrophic malformations, and drugs.

A key feature of minoxidil, when used to oppose the androgen-dependent genetically programmed progressive hair follicle miniaturization, is to maintain or enhance normal dynamics of the essential stromal-epithelial interaction that occurs between the dermal papilla of the hair follicle and the proliferating hair matrix cells which the papilla serves. Hair matrix is one of the most rapidly proliferating tissues in the human body and in a carefully orchestrated symphony of controlled differentiation produces six different cells lines: the medulla, the cortex, and the cuticle of the hair shaft, as well as the cuticle and the Huxley and Henle layers of the inner root sheath. The presence of the dermal papilla is an absolute requirement for this remarkable differentiation. The matrix epithelium itself is indifferent and will not differentiate in the absence of the papilla. Experimental explants of dermal papillae in rodents have resulted in new hair growth directly from mucous membrane or from the interfollicular epidermis. ${ }^{2,3}$

\section{Histologic Studies}

Histologic studies of human scalps treated with topical minoxidil provide no evidence for follicular neogenesis-the formation of new hair follicles. ${ }^{1}$ New follicle formation would require that minoxidil or similar substances selectively aggregate specialized mesenchyme, that is, result in the formation of new dermal papilla. To date, there is no suggestion that topically applied minoxidil has such an effect. This has been confirmed by counting total hairfollicles per unit area of scalp before and after treatment, as well as by microscopic examination of the outer sheath of follicles and the interfollicular epidermis. ${ }^{4}$

Hair shaft diameters may decrease as much as 10 times in the balding patient-from 0.1 $\mathrm{mm}$ to $0.01 \mathrm{~mm}$. Hair shaft diameter is in all probability, a function of matrix volume. Under the influence of topical minoxidil, small preexisting terminal hair follicles enlarge. This hypertrophic effect includes an increase in hair 
matrix volume. There have been no changes observed in matrix differentiation; the enlarged follicles observed following the use of topical minoxidil are normally structured. Also, there has been no suggestion of disordered or atypical follicular growth within the limits of duration of the clinical studies completed thus far. It is essential that minoxidil and other trichotrophic substances do not perturb normal matrix cell proliferation or differentiation.

The hair shafts formed by miniaturized follicles in androgenetic alopecia are not only thinner than normal, they are also shorter than normal. Short hairs are the result of a shortened anagen time. As yet, it is not known what biologic events other than genetic programming determine the duration of anagen. For a trichotrophic substance such as minoxidil to be cosmetically effective there must be a prolongation of anagen time in order to grow a longer hair. This effect must be accompanied by the promotion of an increased matrix volume for an increased hair shaft diameter-thicker hair.

Although there is no direct proof yet that topical minoxidil prolongs the anagen phase of the hair cycle, there is good circumstantial evidence of this action. Often within the first 90 days after beginning topical minoxidil use, patients observe a decrease in hair loss-less hair falling out by the roots. Histologic confirmation of this is evidenced by decreased telogen counts in posttreatment biopsy samples as compared with pretreatment counts in the same individual. ${ }^{4}$

It is important to note that minoxidil probably does not prolong the anagen phase of the treated follicle indefinitely. Although the duration of anagen may be increased, individual follicles appear to continue to cycle. 'This observation is particularly relevant to the rate at which effective cosmetic benefit is achieved (visible hair growth). It may be necessary, therefore, for a number of consecutive cycles to occur, with only modest increases in length and thickness from each cycle, before maximum potential gain is achieved. The degree to which a trichotrophic substance can maintain the "on" or anagen signal is a very important component of efficacy and undoubtedly is actively opposed by the normal genetic program of the affected individual. The ongoing androgen-dependent genetic program for miniaturization may ultimately prove to produce a tachyphylactic effect.

It is fascinating to observe that the application of an organic molecule to the scalp surface can result in normally structured follicular hypertrophy and prolonged follicular growth. Minoxidil may increase blood flow to the scalp, but it is not known if minoxidil increases blood flow to the biologically critical dermal papilla. It has also recently been determined that minoxidil inhibits prostaglandin $\mathrm{I}^{2}$ formation in cultured human keratinocytes, ${ }^{5}$ inhibits the synthesis of lysyl hydroxylase in cultured human fibroblasts, ${ }^{6}$ and acts as a $\mathrm{K}^{+}$channel agonist in human vascular smooth muscle. ${ }^{7}$ It is not known if these effects are important for hair growth and the precise mechanism for the trichotrophic action of minoxidil remains unknown.

A receptor site for minoxidil in the dermal papilla or in hair matrix epithelium has not yet been discovered, but it is the conclusion of this author that the most likely site of action is the dermal papilla. Whatever may be ultimately determined, the use of topical minoxidil and other trichotrophic substances have given a strong impetus to the study of normal hair biology.

\section{References}

1. Headington JT. Transverse microscopic anatomy of the human sealp. Arch Dermatol. 1984:120:449-456.

2. Kollar $\mathrm{EJ}$. The induction of hair follicles by embryonic dermal papillae. J Invest Dermatol. 1970;55:374-378.

3. Oliver RF. Responses of oral epithelium to the influence of whisker dermal papillae in the adult rat. Arch Oral Biol. 1973;18:413-421.

4. Headington JT. Novak E. Clinical and histologic studies of male pattern baldness treated with topical minoxidil. Cur Ther Res. 1984;36:1098-1106.

5. Baden HP. Selective action of minoxidil on arachidonic acid metabolism of cells in culture. Androgenetic Alopecia: A Brook Lodge Symposium. The Upjohn Company, Kalamazoo, MI, February 16-18. 1987

b. Pinnell SR. Murad S. Effects of minoxidil on human skin fibroblasts in culture. Clin Dermatol. 1988;6(4):152-158.

7. Meisheri KD, Cipkus LA. Minoxidil sulfate produces vasodilation by increasing plasmalemmel $\mathrm{K}^{+}$permeability. Presented at the Brook Lodge Symposium, Androgenetic Alopecia, Kalamazoo, MI February 16-18, 1987 